\title{
Assessment of the Technological and Environmental Factors Influencing the Ultra-High-Pressure Water Jetting Treatment of the Surface of Naval Construction Steel Against Corrosion
}

\author{
${ }^{1}$ Luis Marcos Castellanos-Gonzalez, ${ }^{2}$ Javier A. Castilla Jimenez, ${ }^{3} J u a n$ Jose Cabello Eras \\ ${ }^{4}$ Julio S. Hurtado Marquez, ${ }^{1}$ Leonel Martinez-Diaz and ${ }^{3}$ Alexis Sagastume Gutierrez \\ ${ }^{1}$ Centro De Crecimiento Empresarial, Titular-Investigador, \\ en Ciencias Tecnicas, Universidad Simon Bolivar-Barranquilla, Barranquilla, Colombia \\ ${ }^{2}$ en Ingenieria Naval, Gerente de Proyectos, COTECMAR-Cartagena, Bolivar, Colombia \\ ${ }^{3}$ Titular y Jefe del Departamento de Energia de la, \\ en Ciencias Tecnicas, Universidad de la Costa, Barranquilla, Colombia \\ ${ }^{4}$ Ciencias Basicas, en Ciencias Matematicas, Universidad Tecnologica de Bolivar, \\ Cartagena, Colombia
}

\begin{abstract}
This research defines the best combination of factors (water pressure, the use or non-use of washing with fresh water after process, environmental factors (i.e., temperature, humidity, etc.) and type of epoxy paint) for the high pressure water jetting treatment of naval steels to guarantee the best performance against corrosion. Results show that the epoxy/iron oxide paint had the best performance when: a water-jetting pressure of $275.8 \mathrm{MPa}$ is used, followed by a low pressure freshwater cleaning. Results also show the best results when the process is developed at the highest environmental temperatures and lowest humidity.
\end{abstract}

Key words: Water-jetting, cleaning, surface preparation, coatings, flash rust, corrosion, results, freshwater

\section{INTRODUCTION}

An adequate surface preparation is resquired to improve the adhesion of marine paints, especially on metal surfaces where corrosion (rusting) resistance is important. To this end, SandBlasting (SB) have been extensively applied. However, because of its consequences on both the human health and the environment its use has been reduced (Rosenberg et al., 2006). Consequently, the UltraHigh-Pressure Water-Jtting (UHPWJ) technology in the fast track to become the standard technology of the surface preparation industry to remove paints from the surface of metals. Although, the are some limitations of UHPWJ as compared to SB greater global cost, not generating a new anchor prople and forming a thin oxide layer (flash rust) at the steel surface before applying the prime (Frenzel, 2010).

Towards an improved effciency and competitiveness of UHPWJ different studies have been developed. Special attention has been paid to advancements in the nozzle technology that increased UHPWJ productivity to similar levels as compared to SB. The automation of UHPWJ technology combined with new equipment designs significantly contributed to this end (Schmid, 2005). In fact, introducing semiautomatic systems resulted in over twice of the productivity of handwork operated UHPWJ. There is also some research developed to modify the anchor profile at the steel surface (Teimourian et al., 2010; Knapp and Taylor, 1996; Calve et al., 2011).

The influence of oxides and dissolved salts concentration on the adherence and durability of paints, have been determined showing that a reduction of the flash rust area below $20-30 \%$ has a negligible impact on the primer performance (Kim et al., 2008). A characterization of ash rusted surfaces with pressures over $206 \mathrm{MPa}$ can be found by Ault $(2010,2014)$ including some alternatives to reduce flash rusting. However, the best combination of the working pressure with the environmental parameters and the type of primer that ensure the best coating performance needs to be further discussed.

This study aims at defining the best combination of the technological and environmental factors (water pressure, the or not for a subsequent washing with fresh water, environmental conditions, kind of epoxy coating) that results in the best performance of naval steels in corrosive environments.

Corresponding Author: Luis Marcos, Centro De Crecimiento Empresarial, Titular-Investigador, en Ciencias Tecnicas, Universidad Simon Bolivar-Barranquilla, Barranquilla, Colombia 


\section{MATERIALS AND METHODS}

The study was developed in a shipyard located in the Colombian Caribbean characterized by highly corrosive humid tropical weather. A two-level experimental design including four factors. Tests samples of repainted naval steel, repaired under standard conditions were subjected to a salt Spray Fog Test (SFT), following the ASTM D1654 standard and using the Taguchi $\mathrm{L}_{8}$ orthogonal array (Bierwagen et al., 2003; Yuin and Alan, 1997).

The 8 specimens of repaired naval steel, taken from a ship ongoing major repairs without changing its sample surfaces were used (Fig. 1). The specimen dimensions are: $7.5 \mathrm{~cm}$ length and $15 \mathrm{~cm}$ width. Surface preparation and painting were developed in similar environmental conditions at the shipyard. The UHPWJ machine can provide a maximum pressure of $379.2 \mathrm{MPa}$.

To assess the influence of Visible (VC) and Non-Visible (NV) contamination, temperature and relative humidity, the specimens were processed in two group, one at 7:00 a.m. and the other at 4:00 p.m.

The water pressure is of great importance in the UHPWJ given its influence in the surface contamination after the process is finished. To assess this influence two pressure levels 241.3 MPa because and 275.8 MPa. The lower pressure (i.e., 241.3 MPa) is the most frequent value used in the plant because the human operator suffers less from fatigue in this case. Figure 2 shows the cleaning of 1 specimen by UHPWJ.

After cleaning with the UHPWJ, washing the surface with fresh water at a low pressure $(22 \mathrm{MPa})$ reduces the chloride concentration in surface. Implementing or not this second cleaning introduces an additional factor influencing the presence of contamination in the metal surface which impacts the coating behavior.

Two different two of the most used epoxy paints in shipbuilding industry (i.e., one with iron oxide and another with polyamide) were tested to assess their performance against corrosion after the UHPWJ treatment.

To define the best combination of factors that guarantee the best physicochemical performance against corrosion, a Taguchi $\mathrm{L}_{8}$ orthogonal array is used (Table 1). This design allows to measure at two levels the effect of up to seven control factors including noise factors. The control factors are placed in the first four columns and 5 repetitions for each test sample allowed the variability caused by the presence of a noise factor to be minimized. Furthermore, two stages were implemented first, the levels of the control factors that minimize the variability were defined using the highest signal/noise
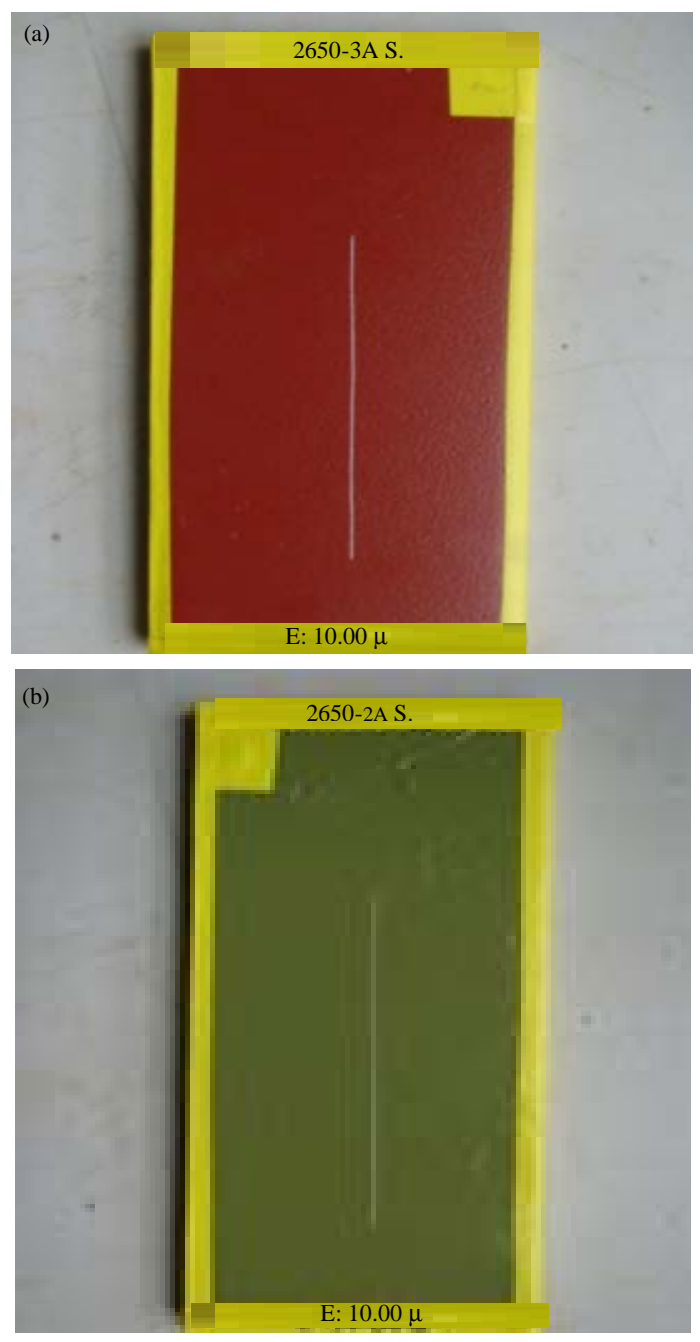

Fig. 1: Specimens readies for corrosion test; a) Coated with epoxy iron oxide and b) Coated with polyamide

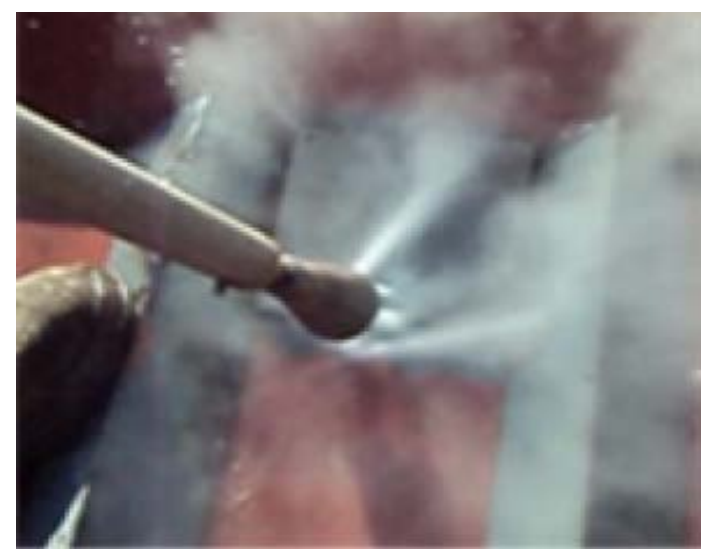

Fig. 2: UHPWJ surface cleaning 
(a)

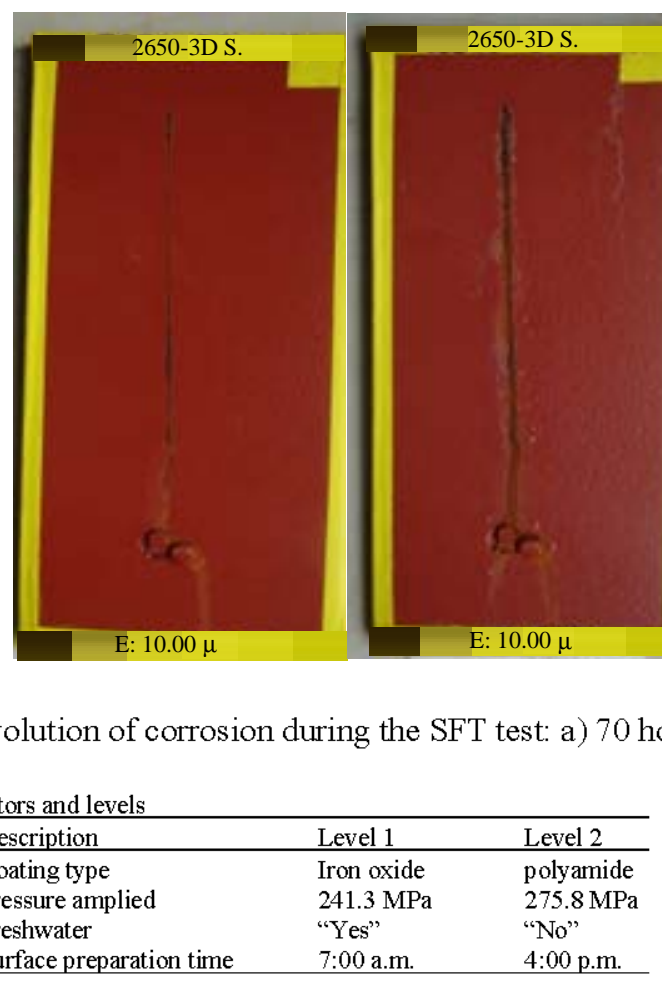

Table $2: L_{8}$ orthogonal array for the experimental study

\begin{tabular}{lllllll}
\hline $\mathrm{A}$ & $\mathrm{B}$ & $\mathrm{C}$ & $\mathrm{D}$ & $\mathrm{e}$ & $\mathrm{e}$ & $\mathrm{e}$ \\
\hline 1 & 1 & 1 & 1 & 1 & 1 & 1 \\
1 & 1 & 1 & 2 & 2 & 2 & 2 \\
1 & 2 & 2 & 1 & 1 & 2 & 2 \\
1 & 2 & 2 & 2 & 2 & 1 & 1 \\
2 & 1 & 2 & 1 & 2 & 1 & 2 \\
2 & 1 & 2 & 2 & 1 & 2 & 1 \\
2 & 2 & 1 & 1 & 2 & 2 & 1 \\
2 & 2 & 1 & 2 & 1 & 1 & 2 \\
\hline
\end{tabular}

ratio (which are achieved at the levels that maximize signal-to-noise); second, the control factor levels that maximize the mean value were defined. The best combination of factors result from the levels that minimize the variability and maximize the mean. The statistical processing was developed in the MINITAB 141 Software. Table 2 shows the "L $L_{8}$ " orthogonal array for the control factors and their levels. Columns 6-8 indicate the random error.

The response variables evaluate the progress of corrosion: the formation of blisters because of corrosion, the loss of adhesion along a cut line or any other issue of a particular epoxy paint resulting from the attack of corrosion by means of Salt Fog Test (SFT) during 50, 150, 300 and $500 \mathrm{~h}$ (Schmid, 2005) performed under ASTM D1654 standard. This tests assess the physicochemical behavior of steel with an atomizer spray a fog with a concentration of $5 \% \mathrm{NaCl}$ by weight at $35^{\circ} \mathrm{C}$ over the steel specimens. Corrosion is evaluated at 10 levels (improving for $0-10$ ) according the SFT test. Figure 3 shows the (c)

(d)

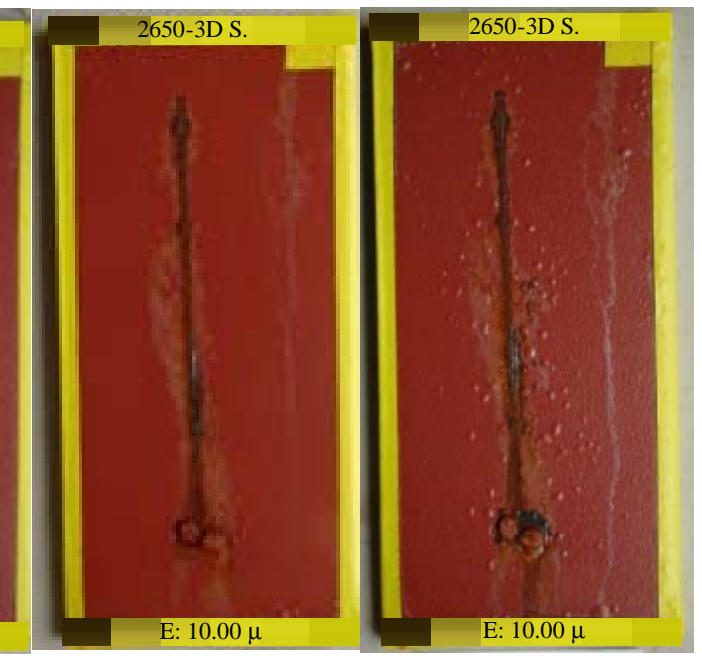

Fig. 3: Evolution of corrosion during the SFT test: a) 70 horas; b) 150 horas; c) 300 horas and d) 500 horas

evolution of the coating failure caused by the accelerated corrosion in one specimen painted with epoxy iron oxide.

\section{RESULTS AND DISCUSSION}

Table 3 shows the $\left(\mathrm{L}_{8}\right)$ orthogonal array of the statistical assessment of results from the SFT. In the assessment, the parameters with higher value have more influence as show in Fig. $4 \mathrm{a}$ where factor $\mathrm{A}$ (the type of paint) has the highest influence with level 1 (iron oxide-type paint) minimizing the variability. Factors B-D showed lower impacts on the variability. The combination of factors that minimize the variability is $(\mathrm{A}, \mathrm{B}, \mathrm{C}, \mathrm{D})=(1,2,1,2)$.

The assessment of the factors affecting the mean, Fig. $4 \mathrm{~b}$ shows the Factor $\mathrm{A}$ (the paint type) has the highest impact with Level 1 (iron oxide-type paint) maximizing the mean. Factors B-D have little influence on the mean. The combination of factors that maximizes the mean is $(A, B, C$ and $D)=(1,2,1$ and 2$)$.

From the experimental results, the best combination of factors for an optimum UHPWJ surface preparation is to start at $4.00 \mathrm{pm}$ using a water pressure of $275.8 \mathrm{MPa}$ in UHPWJ operation and washing afterwards with freshwater. Additionally, the physicochemical performance of naval steel exposed to corrosive environments improves with the use of iron oxide-based paints.

The results of variance analysis are shown in Table 4 and 5, mainly concluding that factor " $\mathrm{A}$ " has the highest effect on the response variable with a $\mathrm{p}<5 \%$ which confirms the results from Fig. 4.

The performance of anticorrosive coating applied to steels is strongly influenced by the surface preparation, 
Res. J. Applied Sci., 13 (5): 294-298, 2018

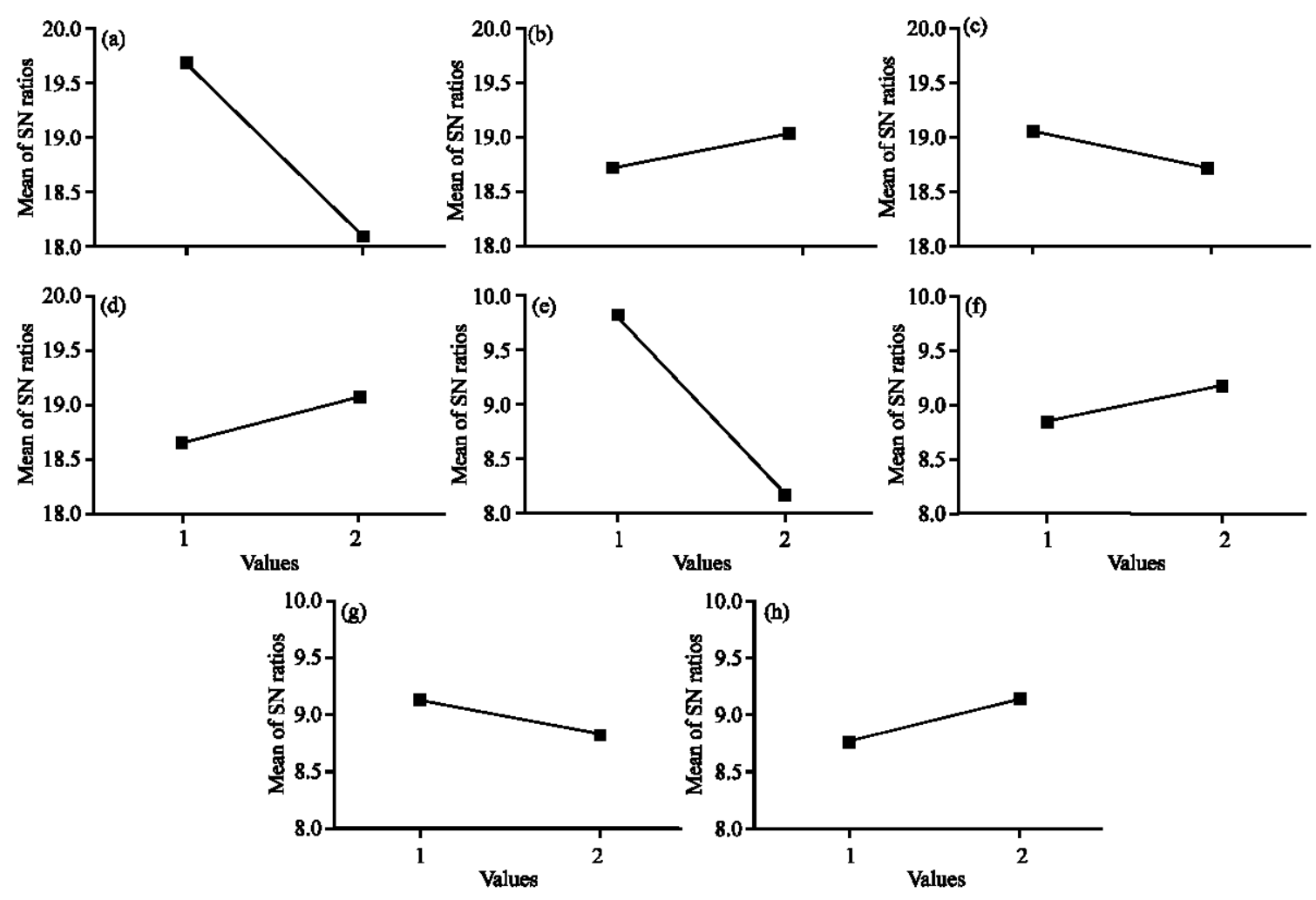

Fig. 4: Factors and interaction; a-d) $\mathrm{S} / \mathrm{N}$ ratios and e-h) The median of the factors

\begin{tabular}{|c|c|c|c|c|c|c|c|c|c|c|c|c|c|}
\hline \multirow{2}{*}{$\begin{array}{l}\mathrm{A} \\
1\end{array}$} & \multirow{2}{*}{$\begin{array}{l}\mathrm{B} \\
1\end{array}$} & \multirow{2}{*}{$\begin{array}{l}\mathrm{C} \\
1\end{array}$} & \multirow{2}{*}{$\begin{array}{l}\mathrm{D} \\
1\end{array}$} & \multirow{2}{*}{$\begin{array}{l}\mathrm{e} \\
1\end{array}$} & \multirow{2}{*}{$\begin{array}{l}\mathrm{e} \\
1\end{array}$} & \multirow{2}{*}{$\begin{array}{l}\mathrm{e} \\
1\end{array}$} & \multicolumn{5}{|c|}{ - } & \multirow{2}{*}{$\begin{array}{l}\mu \\
10\end{array}$} & \multirow{2}{*}{$\frac{\mathrm{S} / \mathrm{N}}{20.00}$} \\
\hline & & & & & & & 10 & 10 & 10 & 10 & 10 & & \\
\hline 1 & 1 & 1 & 2 & 2 & 2 & 2 & 10 & 10 & 10 & 10 & 10 & 10 & 20.00 \\
\hline 1 & 2 & 2 & 1 & 1 & 2 & 2 & 10 & 10 & 10 & 10 & 10 & 10 & 20.00 \\
\hline 1 & 2 & 2 & 2 & 2 & 1 & 1 & 10 & 10 & 10 & 10 & 10 & 10 & 20.00 \\
\hline 2 & 1 & 2 & 1 & 2 & 1 & 2 & 10 & 7 & 7 & 7 & 7 & 7.6 & 17.36 \\
\hline 2 & 1 & 2 & 2 & 1 & 2 & 1 & 10 & 8 & 8 & 7 & 7 & 8.0 & 17.84 \\
\hline 2 & 2 & 1 & 1 & 2 & 2 & 1 & 10 & 8 & 7 & 7 & 7 & 7.8 & 17.60 \\
\hline 2 & 2 & 1 & 2 & 1 & 1 & 2 & 10 & 9 & 9 & 9 & 9 & 9.2 & 19.25 \\
\hline
\end{tabular}

Table 4: Analysis of the $\mathrm{S} / \mathrm{N}$ ratio variance

\begin{tabular}{lcrcccc}
$\begin{array}{l}\text { Source of } \\
\text { variation }\end{array}$ & df & \multicolumn{1}{c}{ Seq SS } & Adj. SS & Adj. MS & F-values & p-values \\
A & 1 & 7.8559 & 7.8559 & 7.8559 & 25.88 & 0.015 \\
B & 1 & 0.3353 & 0.3353 & 0.3353 & 1.10 & 0.370 \\
C & 1 & 0.3353 & 0.3353 & 0.3353 & 1.10 & 0.370 \\
D & 1 & 0.5671 & 0.5671 & 0.5671 & 1.87 & 0.265 \\
Residual error & 3 & 0.9106 & 0.9106 & 0.3035 & - & - \\
Total & 7 & 10.0042 & - & - & - & -
\end{tabular}

optimal environmental conditions and a good primer. The percentage of flash-rust concentration is lower for higher water pressure in UHPWJ and the surface preparation taking place in the afternoon with higher temperature and lower moisture. On the other hand, the chloride concentration slightly decreases (from a mean value of 4.3-3.2 $\mu \mathrm{Lg} / \mathrm{cm}^{2}$ ) when a freshwater washing at low pressure is applied after the UHPWJ. Although, chloride concentrations are acceptable for paint application
Table 5: Analysis of the median variance

\begin{tabular}{lcccccc}
\hline $\begin{array}{l}\text { Source of } \\
\text { variation }\end{array}$ & df & Seq. SS & Adj. SS & Adj MS & F-values & p-values \\
\hline A & 1 & 6.845 & 6.845 & 6.8450 & 31.35 & 0.011 \\
B & 1 & 0.245 & 0.245 & 0.2450 & 1.12 & 0.367 \\
C & 1 & 0.245 & 0.245 & 0.2450 & 1.12 & 0.367 \\
D & 1 & 0.405 & 0.405 & 0.4050 & 1.85 & 0.266 \\
Residual error & 3 & 0.655 & 0.655 & 0.2183 & - & - \\
Total & 7 & 8.395 & - & - & - & - \\
\hline
\end{tabular}

according to NACE No 5/SSPC-SP 12. However, this reduction guarantees a lower absorption of humidity by osmosis through the coating and consequently, less superficial rust is produced.

Table 3 shows that epoxy-based coating with iron oxide has the best performance against corrosion. However, the epoxy paint with polyamide also showed good performance for the same combination of factors: water pressure of $275.8 \mathrm{MPa}$, subsequent 
washing with fresh water and afternoon environmental conditions with highest temperature and lower humidity.

\section{CONCLUSION}

The best combination factors affecting the performance of the anticorrosion coating in naval steels to repair ships was defined. Conclusion points to the use of iron oxide based epoxy paints, highest water pressure and washing at low pressure afterwards. The humidity of the base material is not a troubling factor because evaporation is almost immediate under the work conditions.

\section{REFERENCES}

Ault, J., 2010. Inspection techniques for flash rust formed after waterjetting. Elzly Technology Corporation, Reston, Virginia.

Ault, J., 2014. Performance of coatings over waterjetted surfaces, paint and coatings industry webinars. Paintsquare Mag., 18: 36-45.

Bierwagen, G., D. Tallman, J. Li, L. $\mathrm{He}$ and C. Jeffcoate, 2003. EIS studies of coated metals in accelerated exposure. Prog. Org. Coat., 46: 149-158.
Calve, P.L., J.P. Pautasso and N.L. Bozec, 2011. Characterizing surfaces after UHP waterjetting in new ship construction. J. Protective Coat. Linings, 28: 36-42.

Frenzel, L.M., 2010. How does waterjet cleaning affect the surface and surface preparation?. J. Protective Coat. Linings, 27: 44-56.

Kim, G.R., T.S. Kang, Y.H. Cho and J.M. Han, 2008. Effect of flash rust on protective properties of organic coatings. Proceedings of the Conference and Expo on CORROSION, March 16-20, 2008, NACE International, New Orleans, Louisiana, pp: 1-13.

Knapp, J.K. and T.A. Taylor, 1996. Waterjet roughened surface analysis and bond strength. Surf. Coat. Technol., 86: 22-27.

Rosenberg, B., L. Yuan and S. Fulmer, 2006. Ergonomics of abrasive blasting: A comparison of high pressure water and steel shot. Appl. Ergon., 37: 659-667.

Schmid, R., 2005. Evolution of UHP waterjetting equipment: Surface preparation process found to yield higherproductivity than gritblasting. Met. Finish., 103: 41-60.

Teimourian, H., M.R. Shabgard and A.W. Momber, 2010. De-painting with high-speed water jets: Paint removal process and substrate surface roughness. Prog. Org. Coat., 69: 455-462.

Yuin, W. and W. Alan, 1997. Robust design using taguchi methods. Librería Díaz de Santos, Madrid, Spain. 